\section{Entrevista al Dr. Roberto Elias: conociendo el futuro de la odontología para pacientes especiales}

\author{
Interview with Dr. Roberto Elias: knowing \\ the future of dentistry for special patients
}

\section{Resumen}

En esta entrevista, el Dr. Roberto Elias nos revela cómo el trabajo odontológico con los pacientes especiales fue el principal motor para la fundación de AIOPE (Academia Interamericana de Odontología para Pacientes Especiales). Dicha asociación tiene como principal objetivo desarrollar actividades académicas que apoyan a las instituciones orientadas a la salud y áreas afines, así como plantear protocolos y políticas públicas para mejorar la calidad de vida de las personas con discapacidad. Asimismo, destaca la importancia de compartir las diferentes experiencias entre los especialistas de todo América y la importancia de incorporar asignaturas durante la enseñanza universitaria tanto en pregrado como posgrado para mejorar la salud bucal de las personas con discapacidad. Resalta el trabajo en equipo, así como conocer y escuchar a cada uno de los pacientes especiales, respetando su condición física, mental, sensorial y comportamental para humanizar la profesión odontológica especialmente dedicada a una población vulnerable.

Palabras clave: Atención Dental para Personas con Discapacidades; Salud bucal; Odontología (fuente: DeCS BIREME).

\begin{abstract}
In this interview, Dr. Roberto Elias reveals how dental work with special patients was the main driver for the foundation of AIOPE (Inter-American Academy of Dentistry for Special Patients). The main objective of this association is to develop academic activities that support health-oriented and related institutions, as well as to propose protocols and public policies to improve living conditions of people with disabilities. Likewise, it highlights the importance of sharing different experiences among specialists from all over America and the importance of incorporating courses during university education both in undergraduate and graduate degrees to improve the oral health of people with disabilities. In addition, teamwork stands out, as well as knowing and listening to every disabled patient, respecting their physical, mental, sensorial and behavioral condition in order to humanize the dental profession dedicated to a vulnerable population.
\end{abstract}

Keywords: Dental care for disabled; Oral health; Dentistry (source: MeSH NLM).

\section{Entrevista}

Edith Falconí Salazar 1,a

${ }^{1}$ Asociación Sociedad Peruana de Odonto Estomatología de Pacientes Especiales (ASPODES), Lima, Perú.

${ }^{a}$ Especialista en Estomatología para Pacientes Especiales. Presidenta de ASPODES.

\section{Correspondencia:}

Edith Falconí Salazar

Correo electrónico: litafalconi@gmail.com

Alameda del Corregidor 1271 La Molina, Lima Perú. ORCID: 0000-0003-1271-3887

Conflicto de interés: el autor declara no tener conflicto de interés.

Fuente de financiamiento: autofinanciado.

Recibido: $18 / 12 / 20$

Aceptado: 04/01/21

Publicado: 15/02/21 


\section{Introducción}

El Dr. Roberto Elias, es investigador, docente de 36 años, coordinador de la especialidad de Odontología para Pacientes Especiales y de la especialidad de Cirugía Maxilo facial en la Universidad UNIGRANRIO, Rio de Janeiro-Brasil, es autor de cinco importantes libros científicos de Odontología para pacientes especiales: "Odontología de alto riesgo" año 1995, "Atención a los pacientes de riesgo" año 2000, "Odontología para pacientes especiales" año 2003, "Odontología para pacientes con necesidades especiales una visión clínica” año 2008, "Odontología para pacientes con necesidades especiales de cero a dieciocho años" 2014 y del libro Odontología para pacientes clínicamente comprometidos, que será lanzado en este año. Es fundador, miembro vitalicio y past presidente gestión 2016 - 2019 de la Academia Interamericana de Odontología para Pacientes Especiales (AIOPE) fundada el 8 de Octubre de 2016, con sede en Rio de Janeiro-Brasil; AIOPE fue creada con el fin de contribuir al progreso de la Odontología para pacientes especiales y ciencias afines en América, por lo cual, pertenecer a la Academia es la mayor prestancia académica en odontología para pacientes especiales en Latinoamérica. Desde su fundación AIOPE se ha propuesto tener representantes académicos de cada país, y en los lugares donde aún no exista la especialidad ayudar a capacitar, sensibilizar a los colegas hacia la formación de especialistas en pacientes especiales.

\section{Entrevista}

\section{¿Desde cuándo existe la Especialidad en Brasil?}

Brasil tal vez fue uno de los primeros países en reconocer la Odontología para pacientes con necesidades especiales, después de muchas luchas y gracias a colegas que siempre defendieron la especialidad, hoy tenemos 20 años de la especialidad.

\section{¿Qué fue lo que le motivó a fundar la Academia AIOPE?}

La precariedad en salud oral de los pacientes especiales, hay muy poca formación de estomatólogos en el área profesional. Hay pocas acciones de políticas públicas para mejorar la salud de los pacientes especiales. Según La Organización Mundial de la Salud (OMS), dos terceras partes de la población que tiene discapacidad no reciben atención bucodental; a pesar de la mayor prevalencia que tienen estas personas de padecer enfermedades bucodentales. La diversidad funcional incluye a aquellas personas que presentan una condición que los aleja de la normalidad, sea de orden físico, mental o sensorial, o de comportamiento que requieren atención especial. Por esto, es importante que desde el gremio odontológico sea este público, privado y en especial si es académico, se realicen todo tipo de estudios que permitan evaluar las condiciones orales de estas personas para poder plantear soluciones acordes a su problemática contribuyendo también a que la mayoría de los profesionales de esta ciencia adquieran los conocimientos y habilidades necesarias para brindar una mejor atención en salud a este tipo de pacientes.
¿Cuáles son los fines que promueve la Academia AIOPE?

En AIOPE queremos ser agentes multiplicadores de protocolos e investigaciones en la atención odontológica en la comunidad de pacientes especiales. Uniendo las diferentes asociaciones y ayudándoles cuando lo necesiten. Está compuesto por colegas destacados y profesionales que han demostrado a lo largo del tiempo sus capacidades en la atención de pacientes especiales. "Trabajar con las capacidades remanentes" (HERMAN, 2020), estamos de acuerdo con ese pensamiento y pensamos que el futuro de la Odontología está en saber escuchar al paciente especial y comprender el mundo en el que vive. La Academia Interamericana de Odontología para Pacientes Especiales nació para aportar y sumar conceptos en el cuidado de estos pacientes de manera constante y activa, sin perder nunca su principal filosofía, la humanización.

¿Cuál es la problemática más frecuente que requiere ser atendida por especialistas en estomatología de pacientes especiales?

La poca formación de profesionales en la atención de los pacientes especiales y la creación de proyectos políticos en el área de odontología. Así como la gran necesidad de atención de este grupo de pacientes, pues se acredita que cerca de $23 \%$ de toda la población mundial son representados por los pacientes especiales.

¿Cómo se encuentra el desarrollo de la estomatología de pacientes especiales en Latinoamérica?

Comenzamos a ver la luz al final del túnel, cada vez más profesionales latinoamericanos se unen, ya sea de forma académica o técnica en el desarrollo de protocolos concretos en ayuda de una terapéutica basada en el desarrollo académico, de manera que se agilicen los conceptos de atención.

¿Cómo se puede promover la enseñanza de Odontología para pacientes especiales en pregrado y posgrado?

La formación de pregrado en cursos de Odontología es una herramienta importante, pues muestra a los alumnos un mundo de realidades con el que ellos por lo general no tienen contacto, siendo una experiencia de vida profesional importante y única. En relación a los cursos de posgrado, se hace necesario mejorar tales cursos de forma científica y profesional. Deben ser administrados en instituciones de credibilidad en concordancia con la Medicina basada en evidencias.

¿Qué características principales debe tener el profesional especialista y cuál debe ser la visión hacia el futuro de nuestra especialidad?

Dentro de las características necesarias tenemos el desarrollo de la humanización, el desarrollo técnico y científico. El futuro es extremadamente prometedor, pues con el desarrollo de la medicina se presentarán más pacientes especiales, sea por la longevidad o sea por sobrevida de los pacientes que años atrás fallecían a los 40 años.

¿Cómo la Asociación Sociedad Peruana de Odonto Estomatología para Pacientes Especiales (ASPODES) puede colaborar con la Academia AIOPE para construir una sociedad más inclusiva? 
ASPODES ya contribuye desarrollando un trabajo científico precioso y atendiendo en las comunidades de pacientes especiales. Un ejemplo a ser seguido por otros países que componen la Academia AIOPE.

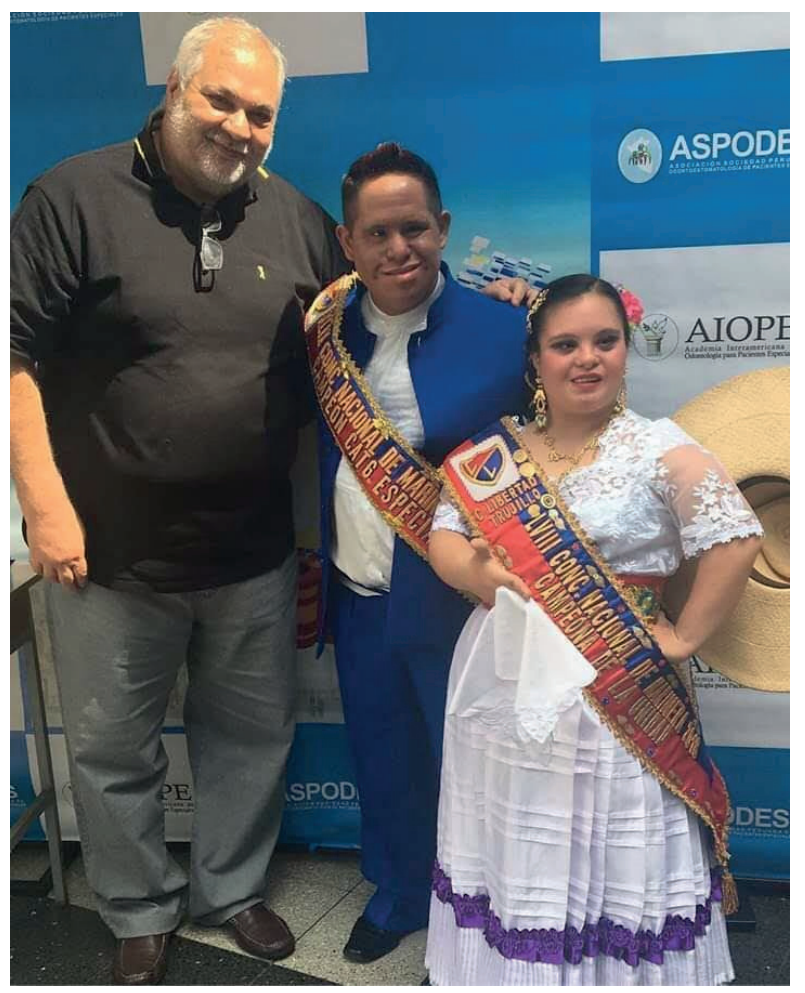

Dr. Roberto Elias durante el Encuentro Científico ASPODES -AIOPE Lima 2018

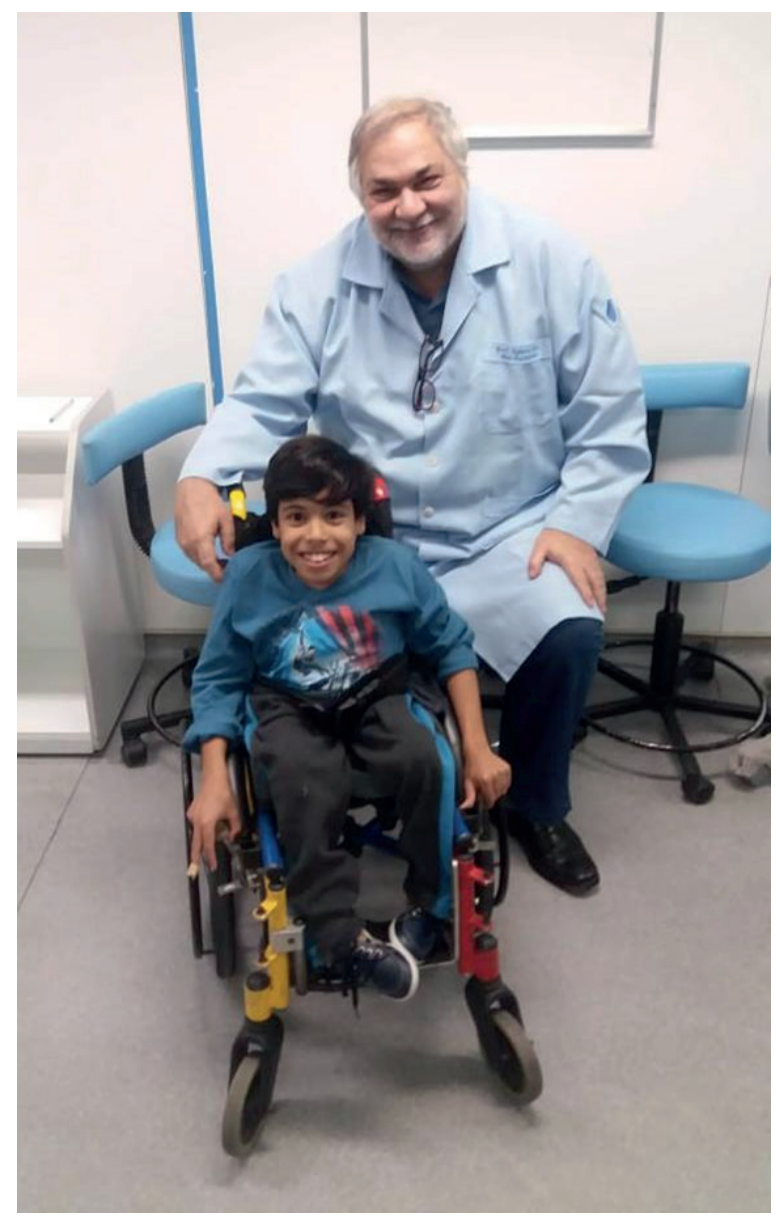

Dr. Roberto Elias demostrando su gran amor por sus pacientes 
